12 Brower K, Eliopulos GA, Blow FC, Catlin DH, Beresford TP. Evidence for physical and psychological dependence on anabolic androgenic steroids in eight weight lifter. $A m \mathcal{F}$ Psychiatry 1990;147:510-2.

13 Bahrke MS, Wright JE, Strauss RH, Catlin DH. Psychological moods and subjectivity perceived behavioural and somatic changes accompanying anabolic-androgenic steroid use. Am F Sports Med 1993:20:717-22.
14 Perry H, Hughes G. A case of affective disorder associated with the misuse of "anabolic steroids". Br $\mathcal{F}$ Sports Med 1992;26:219-20.

15 Reis R. Co-occurance of substance abuse disorders, psychiatric disorders-separation "an art". Psycholink Aug 8 1993.

\title{
Clinical tests in sports medicine: more on Achilles tendon
}

The diagnosis of Achilles tendon rupture can be difficult even for experienced practitioners, and a significant number of patients can be initially misdiagnosed.

When examined soon after the injury, a tear in the substance of the tendon can be seen and palpated. With increased time after the tear, the gap can be obliterated by oedema, and palpation becomes unreliable.

In addition to the tests described in this journal in the June issue (volume 30 , page 124), two other clinical tests should be mentioned.

\section{Matles test}

The patient, lying prone and with feet over the end of the examination couch, is asked to flex the knee actively to $90^{\circ}$. The position of the ankle and feet is observed during this movement. If the foot on the affected side falls into neutral or into dorsiflexion, an Achilles tendon rupture is diagnosed. In normal patients, the foot remains in slight planter flexion when the knee is flexed to $90^{\circ} .{ }^{1}$

\section{Needle test}

With the patient prone, a small gauge needle is inserted at a right angle through the skin of the calf, just medial to the midlline, $10 \mathrm{~cm}$ proximal to the superior border of the calcaneum. The needle should be inserted only until the tip is just within the substance of the tendon. The foot is then passively and alternately dorsiflexed and plantarflexed. Two responses may occur. The needle may swivel so that it points in the direction opposite to the motion of the ankle (that is, when the ankle is dorsiflexed, the needle points distally). This indicates that the tendon is intact in the portion distal to the needle insertion point. The other possible response is that the needle does not move, or moves slightly in the same direction of the motion of the ankle (that is, when the ankle is dorsiflexed, the needle points proximally). In this second instance, the Achilles tendon has lost its continuity between the needle and its insertion. ${ }^{2}$

\section{More on the calf squeeze test}

Given the history of the test, the correct denomination should be Simmond's test. Simmonds described his test in a paper on the diagnosis of Achilles tendon rupture, published five years before Thompson's papers. ${ }^{3}$ Thompson and Doherty published jointly an article in an American journal solely on this test in $1962 .{ }^{4}$ Practically the same paper was published by Thompson, without his colleague, some months later in a European journal. ${ }^{5}$

In the British Isles, the calf squeeze test is called "Simmond's test", and in most of the rest of the world "Thompson's test", not acknowledging that Doherty was one of the original authors. To avoid offending the sensitivities of British or American influenced surgeons, it is better to describe the test as the "calf squeeze test" and cite the fact that Simmonds described it well before Thompson and Doherty. To avoid confusion on what constitutes a positive test, one should probably just mention whether the ankle plantar flexes on squeezing the calf muscles. If it does, the tendon is most likely intact (but beware of the plantaris!).

NICOLA MAFFULLI

1 Matles AL Rupture of the tendo Achilles. Another diagnostic test. Bull Hosp foint Dis 1975;36:48-51.

O'Brien T The needle test for complete rupture of the Achilles tendon. 7 Bone foint Surg Am 1984;66:1099-101 Achilles tend Simmonds FA The diagnosis of

tendon. Practitioner 1957;179;56-8 Thompson TC, Doherty JC. Spontaneous rupture of the Achilles tendo

1962;2:126-9. A test for rupture of the tendo Achillis. Acta Orthop Scand 1962;32:4615. 